\title{
Biological basis for efficacy of activin receptor ligand traps in myelodysplastic syndromes
}

\author{
Amit Verma, ${ }^{1}$ Rajasekhar N.V.S. Suragani, ${ }^{2}$ Srinivas Aluri, ${ }^{1}$ Nishi Shah, ${ }^{1}$ Tushar D. Bhagat, ${ }^{1}$ Mark J. Alexander, ${ }^{2}$ Rami Komrokji, ${ }^{3}$ \\ and Ravi Kumar ${ }^{2}$
}

'Albert Einstein College of Medicine, Montefiore Medical Center, Bronx, New York, USA. ${ }^{2}$ Acceleron Pharma, Cambridge, Massachusetts, USA. ${ }^{3}$ Moffitt Cancer Center, Tampa, Florida, USA.

\begin{abstract}
Signaling by the TCF- $\beta$ superfamily is important in the regulation of hematopoiesis and is dysregulated in myelodysplastic syndromes (MDSs), contributing to ineffective hematopoiesis and clinical cytopenias. TCF- $\beta$, activins, and growth differentiation factors exert inhibitory effects on red cell formation by activating canonical SMAD2/3 pathway signaling. In this Review, we summarize evidence that overactivation of SMAD2/3 signaling pathways in MDSs causes anemia due to impaired erythroid maturation. We also describe the basis for biological activity of activin receptor ligand traps, novel fusion proteins such as luspatercept that are promising as erythroid maturation agents to alleviate anemia and related comorbidities in MDSs and other conditions characterized by impaired erythroid maturation.
\end{abstract}

\section{Introduction}

Transforming growth factor- $\beta$ (TGF- $\beta$ ) superfamily signaling is important in the regulation of hematopoiesis through effects on cell quiescence, apoptosis, proliferation, differentiation, and migration (1-5). Canonical signaling by ligand-receptor complexes in this superfamily is mediated intracellularly by SMADs, which form two pathway branches comprising SMAD2/3 and SMAD1/5/8 (6). Under normal conditions, SMAD2/3-pathway ligands such as activins and growth differentiation factors (GDFs) exert inhibitory regulatory effects on multiple phases of erythropoiesis $(5,7,8)$. However, under certain pathologic conditions this pathway can become dysregulated, leading to anemia $(7,9,10)$. Through their ability to reduce SMAD $2 / 3$ signaling $(7,10)$, activin receptor ligand traps such as luspatercept and sotatercept alleviate anemia in patients with myelodysplastic syndromes (MDSs) and $\beta$-thalassemia $(11,12)$, thus demonstrating the relevance of SMAD2/3 signaling in these diseases characterized by ineffective erythropoiesis. In this Review, we describe myelosuppressive signaling pathways whose activation in MDSs leads to anemia. We also describe the biological basis of activity of activin receptor ligand traps and the role of SMAD proteins in their efficacy.

\section{Ineffective erythropoiesis causes anemia in MDSs}

MDSs comprise a heterogeneous group of clonal bone marrow disorders characterized by impaired hematopoiesis resulting in cytopenias. Given the heterogeneity in MDS symptoms and the risk of progression to acute myeloid leukemia, treatment

Conflict of interest: R Kumar, RNVSS, and MJA are employees of, and own equity in Acceleron Pharma. AV has received research funding from GlaxoSmithKline, Incyte, MedPacto, Novartis, Curis and Eli Lilly and Company, has received compensation as a scientific advisor to Novartis, Stelexis Therapeutics, Acceleron Pharma, and Celgene, and has equity ownership in Stelexis Therapeutics.

Copyright: $\odot 2020$, American Society for Clinical Investigation.

Reference information: J Clin Invest. 2020;130(2):582-589.

https://doi.org/10.1172/JCl133678. approaches vary for individual patients based on risk stratification systems (13). However, anemia is the defining characteristic in most patients with MDS, being present in approximately $85 \%$ of MDS patients at diagnosis or during the course of the disease (14).

Steady-state erythropoiesis, the normal pathway for production of red blood cells, is a complex process consisting of early and late stages, which in turn comprise a series of phases, as shown in Figure 1. Early-stage erythropoiesis refers to the proliferation of pluripotent hematopoietic stem cells and their differentiation into erythroid progenitors - erythroid burst-forming units (BFU-E) and erythroid colony-forming units (CFU-E) - that in turn generate proerythroblasts. Late-stage erythropoiesis encompasses a process known as terminal erythroid differentiation in which proerythroblasts undergo four or five rounds of mitosis to generate enucleated reticulocytes and finally red blood cells (Figure 1). During the late stage, changes in the morphology of erythroid precursors (erythroblasts) are dramatic and include nuclear condensation, reduction in cell size, and eventually nuclear extrusion.

Ineffective erythropoiesis is defined operationally by the inability to produce an adequate number of red blood cells despite the presence of increased numbers of immature erythroid precursors (15). This condition can arise from either congenital or acquired impairments in erythroid maturation. Molecular mechanisms responsible for ineffective erythropoiesis have been studied intensively in $\beta$-thalassemia (15), a congenital disease in which a quantitative defect in the synthesis of $\beta$-globin chains causes accumulation of free $\alpha$-globin chains that form toxic aggregates in erythroid precursors. In MDSs, in which impaired terminal erythroid differentiation is a strong prognostic indicator of overall survival (16), key defects implicated in dyserythropoiesis (which leads to ineffective erythropoiesis) include disruption of erythroid nuclear opening and histone release as well as reduced levels of GATA-1, a master regulator of erythroid differentiation $(17,18)$. Thus, treatments for anemia resulting from ineffective erythropoiesis will likely require agents with efficacy against multiple, and potentially diverse, causes of impaired erythroid maturation. 


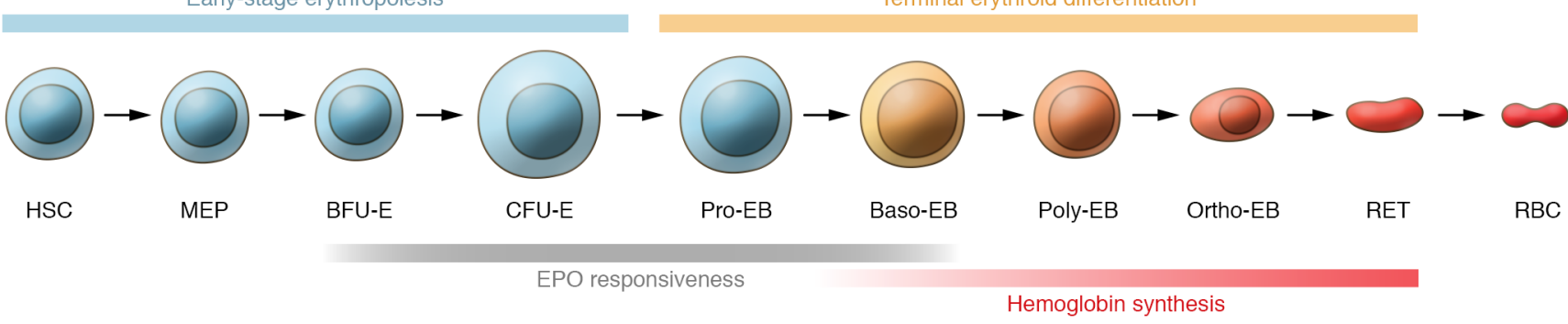

Figure 1. Erythroid differentiation pathway. Pathway starting with an uncommitted hematopoietic stem cell (HSC) and leading to the first committed erythroid progenitor cell (burst-forming unit-erythroid, BFU-E). This marks the start of erythroblast proliferation followed by distinct phases of terminal erythroid differentiation to produce mature red blood cells (RBC). The pathway is depicted as linear for simplicity, but cellular proliferation at early stages amplifies red cell production. MEP, bipotent megakaryocytic-erythroid progenitor; CFU-E, colony-forming unit-erythroid; Pro-EB, proerythroblast; Baso-EB, basophilic erythroblast; Poly-EB, polychromatophilic erythroblast; Ortho-EB, orthochromatic erythroblast; RET, reticulocyte; EPO, erythropoietin. Adapted with permission from Nature Reviews Nephrology (56).

\section{Overview of TCF- $\beta$ superfamily signaling in hematopoiesis}

Dimeric ligands in the TGF- $\beta$ superfamily exert effects by triggering formation of heteromeric complexes containing two type I and two type II receptors belonging to the superfamily $(6,19)$. In some cases, an accessory protein (e.g., betaglycan, endoglin, or Cripto) modulates ligand-receptor interactions in a context-dependent manner but lacks kinase activity and is therefore referred to as a coreceptor $(20,21)$. Type I and type II receptors are single-pass transmembrane proteins containing serine/threonine kinase activity in their distinct intracellular domains. Ligand-mediated activation of these receptors causes phosphorylation of regulatory SMADs (R-SMADs) such as SMAD2 or SMAD3, binding of an
R-SMAD pair to the co-SMAD protein SMAD4, and translocation of this trimeric SMAD complex to the nucleus, where it binds to chromatin and alters expression of target genes (ref. 22 and Figure 2). Importantly, activity in this pathway can be regulated by feedback from inhibitory SMADs (I-SMADs) such as SMAD7 (23). Besides the foregoing canonical pathway, TGF- $\beta$ superfamily ligands can activate non-SMAD pathways such as MAP kinases (p38, ERK, and JNK), Rho-like GTPase, and PI3K/AKT (24).

A key feature of TGF- $\beta$ superfamily signaling is the promiscuous interaction of its members (25). This arrangement allows cells to perceive information encoded in combinations of ligands (26), including context-dependent antagonism of low-affinity ligands by high-affinity ones (27). TGF- $\beta$ superfamily ligands can be clas-

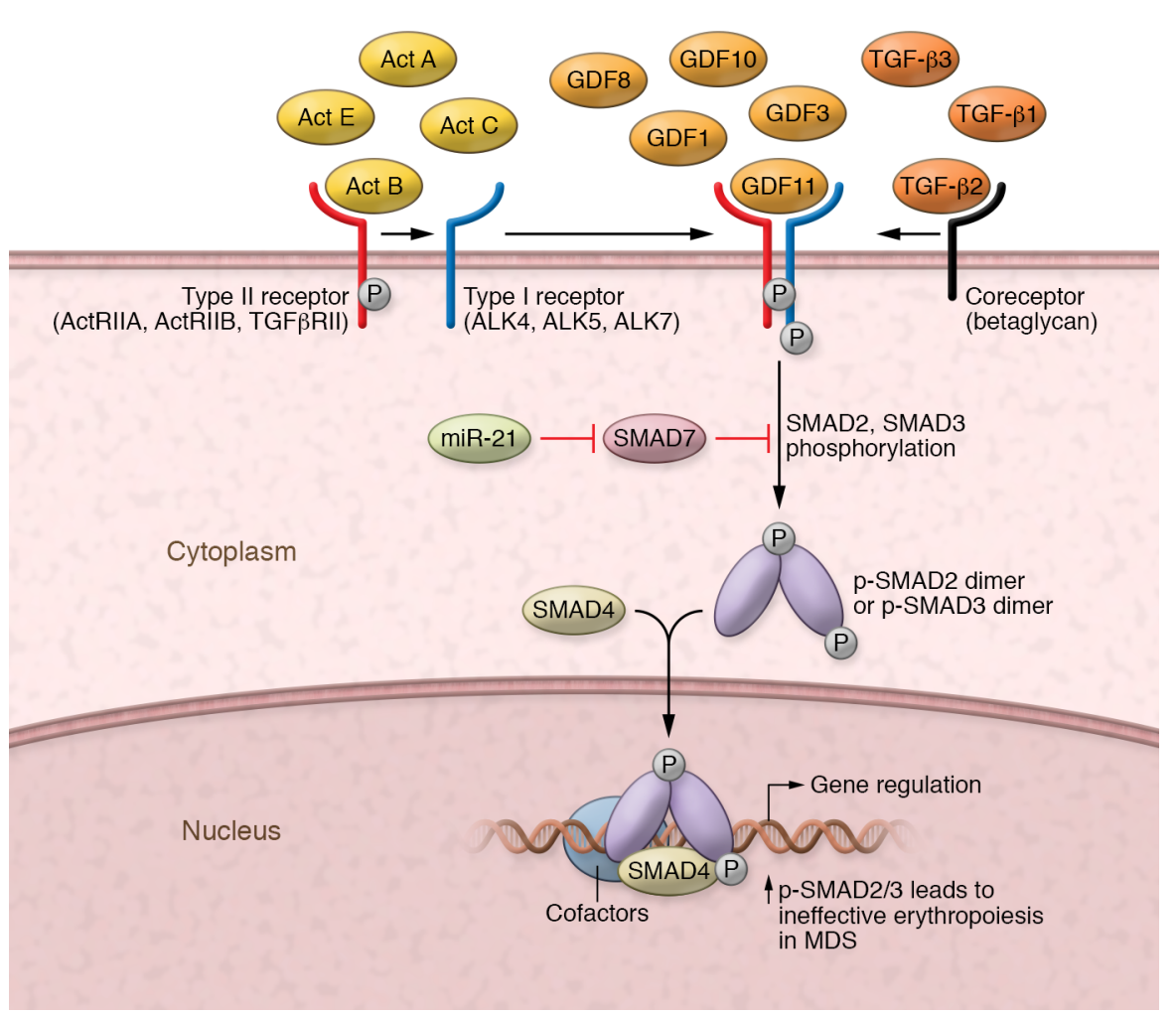

Figure 2. Canonical signaling by SMAD2/3pathway ligands. Ligand binding leads to multimerization of type I and type II receptors, in some cases with the assistance of a coreceptor (type III). Activated type I receptors phosphorylate SMAD2 or SMAD3, which dissociate from type I receptor and oligomerize with SMAD4 to form a heterodimeric complex that translocates into the nucleus, thereby regulating the cellular response. SMAD7, whose stability is regulated by microRNAs (miR-21), can exert feedback effects on the pathway through multiple mechanisms, including inhibition of SMAD2/3 activation. See text for details. While TGF- $\beta$, GDF11, and activin $B$ have been implicated in ineffective hematopoiesis, additional SMAD2/3-pathway ligands are likely involved. Note that dimeric ligands and receptors are depicted here as monomers for simplicity. ALK4/5/7, activin receptor-like kinases 4,5 , and 7. 
A Ineffective erythropoiesis

Hyperproliferation

Elevated SMAD2/3 ligand levels

Impaired erythroid maturation

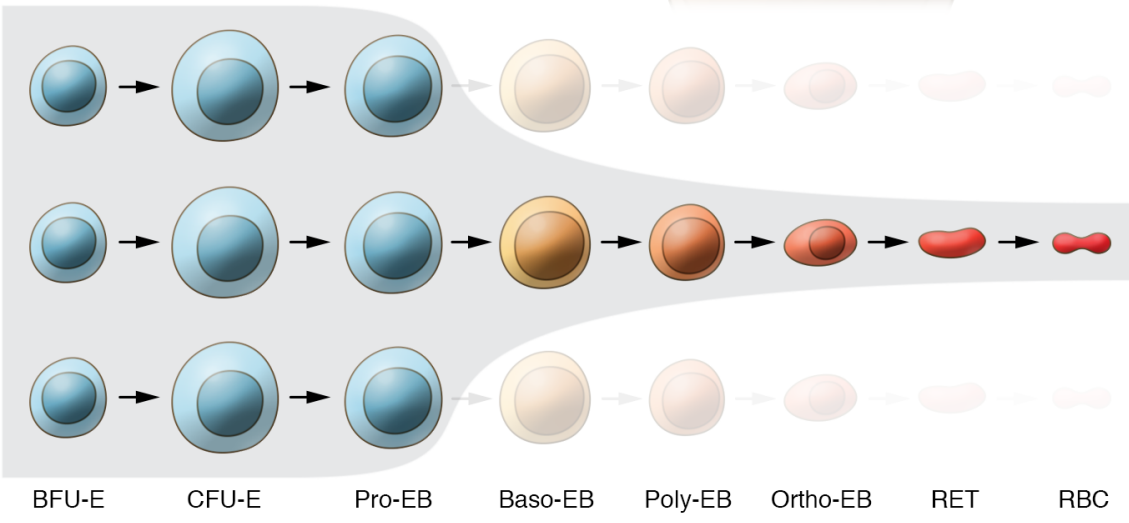

B

Luspatercept therapy

Trapped SMAD2/3 ligands

Reduced SMAD2/3 signaling

Restored erythroid maturation

Effective erythropoiesis
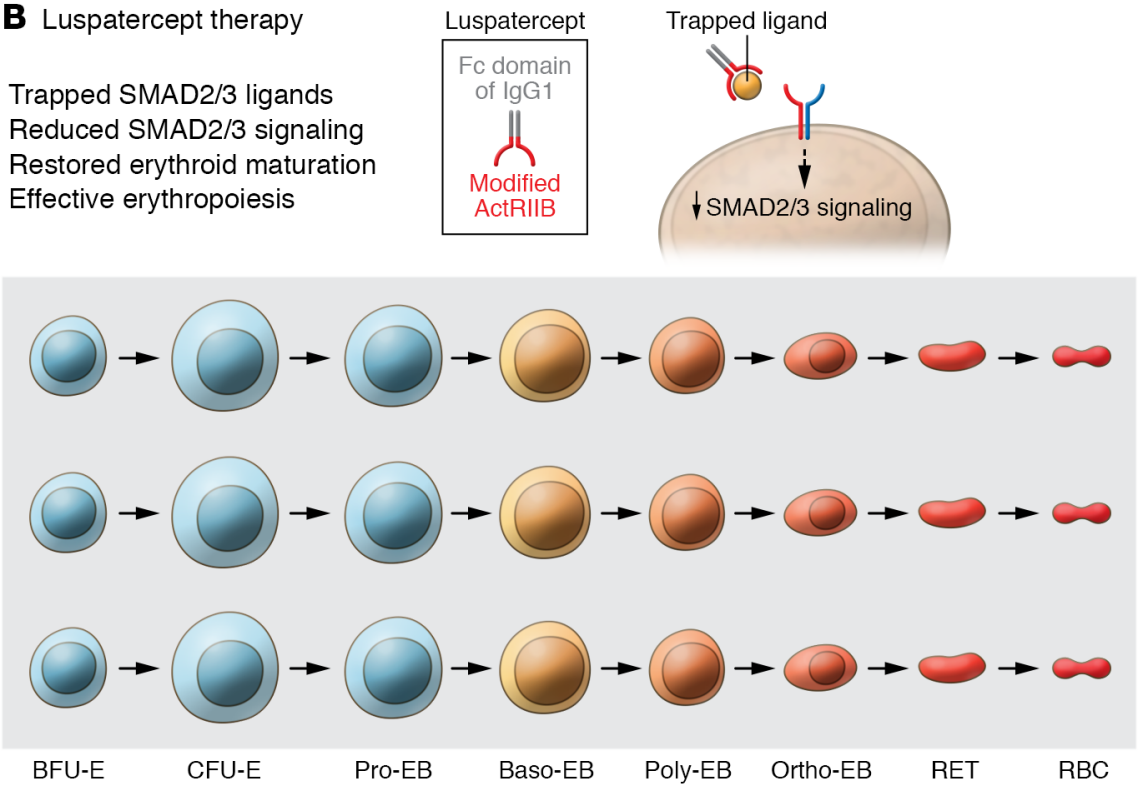

Figure 3. Schematic depiction of SMAD2/3 ligand trapping by luspatercept to treat impaired erythroid maturation and alleviate ineffective erythropoiesis. (A) Ineffective erythropoiesis is the inability to produce an adequate number of red blood cells despite the presence of increased numbers of immature erythroid precursors driven by elevated EPO in response to general hypoxia. Elevated levels of SMAD2/3 signaling contribute to impaired erythroid maturation. (B) Sequestration of SMAD2/3-pathway ligands by therapeutic treatment with activin receptor-based traps such as luspatercept can restore erythroid maturation and alleviate anemia.

sified according to which specific R-SMAD they trigger. Ligands such as TGF- $\beta$, activins, GDF11, and GDF8 signal primarily through SMAD2/3 (Figure 2), while bone morphogenetic proteins (BMPs) and many GDFs signal through SMAD1/5/8 (25). These two branches of the canonical pathway tend to exert opposing effects in cells or tissues, and an imbalance of activity in the two branches is thought to underlie certain pathologic conditions (28).

SMADs are important physiologic regulators of hematopoiesis. SMAD2/3 involvement is best exemplified by the prominent role of TGF- $\beta$ in quiescence and self-renewal of hematopoietic stem cells as well as its ability to stimulate and/or inhibit proliferation of specific cell lineages $(29,30)$. As discussed in detail below, studies with activin receptor ligand traps have revealed that other SMAD2/3 ligands regulate erythroid maturation under normal conditions and contribute to dysregulated erythroid maturation under pathologic conditions. Studies in mice and zebrafish implicate SMAD1/5/8 signaling in particular aspects of hematopoiesis and erythropoiesis $(30,31)$. Activated R-SMADs exert their effects in the nucleus by forming complexes with site-specific transcription factors and binding to enhancer or promoter sequences in the regulatory region of target genes, thereby activating or repressing transcription. Activated R-SMAD complexes also regulate transcription by recruiting coactivators and corepressors that modify histones and/or chromatin structure (22).

Recent studies have helped to clarify the role of TGF- $\beta$-mediated signaling in normal and dysregulated hematopoiesis. In hematopoietic stem cells, an essential role for the superfamily coreceptor endoglin has been identified in promoting TGF- $\beta$ signaling to ensure maintenance of stem cell quiescence (32). Additionally, a downstream target of TGF- $\beta$-mediated SMAD2/3 signaling is responsible for keeping intrinsically motile hematopoietic stem cells in the stromal niche of bone marrow (32). In BFU-E erythroid progenitors, transient elevation of the coreceptor betaglycan promotes TGF- $\beta$ signaling, which in turn reduces self-renewal of these progenitors and total erythroblast production (33). Furthermore, overactivation of TGF- $\beta$ signaling mediated by SMAD $2 / 3$ has been implicated as a cause of bone marrow failure in Fanconi anemia and ShwachmanDiamond syndrome, both disorders with MDS predisposition $(34,35)$. These recent findings underscore the important role of TGF- $\beta$ signaling at early steps in hematopoiesis and in early-stage erythropoiesis, which raises the question of whether SMAD2/3-pathway ligands also participate in the regulation or dysregulation of late-stage erythropoiesis.

\section{SMAD2/3 activation contributes to ineffective erythropoiesis in MDSs}

In three interrelated studies, we have investigated involvement of TGF- $\beta$ superfamily signaling in the dysregulated hematopoiesis that characterizes MDSs. To determine the role of SMAD pathways in this disease, we first examined SMAD activation 
status in primary bone marrow samples from patients with MDS. Compared with controls, MDS patient bone marrow contained higher numbers of bone marrow cells with activated (phosphorylated) SMAD2 (p-SMAD2), as well as greater intensity of p-SMAD staining, thus demonstrating that SMAD2 is constitutively activated and overexpressed in hematopoietic precursors from MDS patients (36). In addition, erythropoiesis was enhanced in a variety of MDS subtypes in vitro when SMAD2 activation was reduced by inhibition of the type I receptor ALK5, either through shRNA-mediated downregulation or pharmacologic inhibition using the small-molecule inhibitor SD-208. Pharmacologic inhibition of ALK5 also alleviated anemia and stimulated hematopoiesis in a mouse model of bone marrow failure, thereby implicating the SMAD2/3 pathway as a potential therapeutic target in MDSs (36).

To probe the mechanistic basis for SMAD2/3 overactivation in MDSs, we next performed a large meta-analysis to determine whether expression of TGF- $\beta$ superfamily genes might be consistently altered in marrow-derived $\mathrm{CD} 34^{+}$cells from MDS patients. This analysis revealed that levels of SMAD7, an important negative-feedback regulator of superfamily signaling, are markedly reduced in MDS in comparison with normal healthy controls (37). SMAD7 can associate with the type I receptor ALK5 and interfere with interactions between ALK5 and SMAD2/3 (Figure 2), thus acting as an endogenous brake on SMAD2/3 pathway activity, and SMAD7 has been found to promote self-renewal of hematopoietic stem cells in vivo (38). Reduced expression of SMAD7 was confirmed in hematopoietic cells, leading to overactivation of SMAD2 signaling despite low concentrations of TGF- $\beta$ (37). Importantly, the small-molecule ALK5 inhibitor LY-2157299 (galunisertib) (a) inhibited TGF- $\beta$-mediated SMAD2 activation and hematopoietic suppression in primary hematopoietic stem cells, (b) alleviated anemia in a transgenic mouse model of bone marrow failure, and (c) stimulated hematopoiesis in bone marrow specimens from MDS patients (37). These results indicate that stem cells and/or progenitors in patients with MDS display overactivated signaling by SMAD2/3, due at least partly to reduced levels of SMAD7, and further indicate that this pathway could be a potential target for therapeutic intervention with ALK5 inhibitors such as galunisertib.

In a third study, we sought to determine the cause of reduced SMAD7 levels in patients with MDS. This study did not find evidence that MDS patients possess a deletion in the $S M A D 7$ locus or aberrant methylation of its promoter. However, we determined that the 3'-UTR of the SMAD7 gene contains a highly conserved, putative binding site for microRNA-21 (miR-21) (39), a molecule upregulated in many disease states and considered to be a key switch in the inflammatory response (40). Levels of miR-21 were elevated in marrow samples of MDS patients (both low-risk and high-risk cohorts) compared with age-matched controls, and miR-21 was revealed to bind directly to the 3'-UTR of SMAD7 and reduce its expression in hematopoietic cells (39). Finally, treatment with a chemically modified inhibitor of miR-21 increased SMAD7 expression in samples from MDS patients in vitro, increased erythroid colony formation from primary MDS bone marrow progenitors in vitro, and increased red cell counts in a mouse model of bone marrow failure arising from TGF- $\beta$ overexpression (39). These results implicate miR-21 as an endogenous regulator of SMAD7 levels, thereby promoting overactivation of SMAD2/3 and ineffective erythropoiesis in MDSs (Figure 2).

Together, the findings reviewed above indicate that reduced SMAD7 levels and constitutive overactivation of SMAD2/3 are novel molecular changes leading to ineffective erythropoiesis in MDS patients. This implies that the SMAD2/3 pathway could be a potential target for therapeutic intervention with small-molecule ALK5 inhibitors or activin receptor ligand traps to alleviate anemia. In a phase II study, galunisertib was associated with hematologic improvements in patients with lower- and intermediate-risk MDS and those heavily dependent on transfusions, but this agent did not meet the primary efficacy endpoint to trigger phase III development (41).

\section{Activin receptor ligand traps as SMAD2/3 signaling inhibitors}

Therapeutic agents that correct overactivated SMAD2/3 signaling by sequestering SMAD2/3-pathway ligands other than TGF- $\beta$ have been developed to treat anemia associated with MDSs and other hematologic diseases. One such agent is luspatercept (ACE536), a ligand-trapping fusion protein containing a modified extracellular domain of activin receptor type IIB (ActRIIB) attached to the Fc domain of human IgG1 (modified ActRIIB-Fc). Structurally related sotatercept (ACE-011) is a ligand-trapping fusion protein containing the extracellular domain of activin receptor type IIA (ActRIIA) attached to the Fc domain of human IgG1 (ActRIIA-Fc). Under cell-free conditions, sotatercept binds activins A and B, GDF8 and GDF11, and some BMPs (BMP6, BMP7, and BMP10) with a range of affinities, reflecting the binding profile of native ActRIIA (27). Luspatercept resembles sotatercept in binding GDF8, GDF11, and activin B with high affinity under cell-free conditions but differs from sotatercept - and native ActRIIA - in part owing to substantially reduced affinity for activin A (7, 42). Importantly, neither luspatercept nor sotatercept binds TGF- $\beta 1$, TGF- $\beta 2$, or TGF- $\beta 3$. Thus, while luspatercept and sotatercept exhibit ligand-binding profiles that overlap considerably, luspatercept displays greater ligand selectivity, which may be advantageous in the context of treating anemia.

These activin receptor ligand traps have also been evaluated in cell-based reporter gene assays. Under such conditions, luspatercept and sotatercept are both able to inhibit phosphorylation of endogenous SMAD2/3, but not endogenous SMAD1/5/8, caused by cell incubation with exogenous ligands $(7,42)$. These results confirm that such agents can effectively compete with endogenous cell surface receptors to inhibit SMAD2/3 signaling caused by TGF- $\beta$ superfamily ligands.

\section{Activin receptor ligand traps in preclinical anemia models}

Activity of luspatercept and sotatercept has been assessed preclinically using the fully human fusion proteins or using murine analogs - RAP-536 or RAP-011, respectively - in which the human IgG1 Fc domain is replaced by its mouse IgG2a counterpart. Luspatercept (or RAP-536) was found to increase red cell counts, hemoglobin concentrations, and hematocrit in nor- 
mal mice, rats, and monkeys in a dose-dependent fashion without altering counts of other blood cells (7). Mechanistic studies indicate that luspatercept increases these red cell parameters by enhancing maturation of late-stage erythroblasts, without altering red cell lifespan appreciably. This mechanism of action is distinct from that of erythropoietin (EPO), which increases red cell counts mainly by increasing proliferation of erythroid progenitor cells (43). The mechanistic distinction is underscored by the synergistic effect of EPO and luspatercept cotreatment in mice (7). Evidence was also obtained implicating SMAD2/3 signaling as an important endogenous inhibitor of erythroid maturation. In rodent models of anemia involving either acute blood loss, chemotherapy-induced anemia, or anemia of chronic kidney disease, animals treated with RAP-536 displayed faster hematologic recovery compared with vehicle-treated controls (7). These findings indicate that luspatercept can increase red cell counts and hemoglobin concentrations under diverse physiologic conditions, including multiple models of anemia.

Importantly, RAP-536 has been evaluated for efficacy against anemia in models of two diseases that are characterized by ineffective erythropoiesis. RAP-536 was first tested in a mouse model of MDS generated by transgenic expression of the NUP98/ HOXD13 (NHD13) fusion protein found in human MDSs and acute myeloid leukemia. These NHD13 mice are characterized by abortive precursor maturation and ineffective hematopoiesis, including ineffective erythropoiesis (44). In NHD13 mice, luspatercept inhibited SMAD2/3 activation, mitigated ineffective erythropoiesis, and ameliorated anemia at multiple stages of disease severity (7). Consistent with increased red cell parameters and reduced anemia, NHD13 mice treated with RAP-536 displayed reduced erythroid hyperplasia and improvement in the abnormal myeloidto-erythroid ratios in the bone marrow (7).

Additionally, RAP-536 was found to ameliorate anemia and improve disease comorbidities in a murine model of $\beta$-thalassemia. RAP-536 treatment inhibited SMAD2/3 signaling in spleen tissue from thalassemic mice, increased red cell counts and hemoglobin concentrations, reduced reticulocytosis and spleen size, and normalized iron stores $(7,9)$. Furthermore, bone mineral density was increased in mice treated with RAP536 , likely as a result of decreased extramedullary erythropoiesis, a known complication of $\beta$-thalassemia. Together, these findings demonstrate the ability of the activin receptor trap luspatercept (RAP-536) to treat anemia secondary to impaired erythroid maturation in models of two different diseases characterized by ineffective erythropoiesis (Figure 3 ). They also implicate the SMAD2/3 pathway as an important inhibitory regulator of erythroid maturation.

As discussed earlier, SMAD2/3 signaling is regulated by multiple ligands of the TGF- $\beta$ superfamily, including activins, GDFs, and TGF- $\beta$. Suragani and coworkers observed that experimental stimulation of SMAD2/3 signaling by administration of GDF11 caused impaired erythroid maturation and anemia in wild-type mice (7). These investigators then used neutralizing antibodies against SMAD2/3-pathway ligands to investigate ligand involvement in erythroid maturation under normal conditions. Importantly, a combination of antibodies against GDF8, GDF11, and activin B was significantly more effective than antibodies against single ligands at stimulating red cell production in wild-type mice, albeit not as effective as RAP536. Consistent with these findings, neither neutralizing antibody against activin A nor global knockout of Inhbc (activin C) or Inhbe (activin E) was able to stimulate red cell production in mice (7). Recent results have been obtained by Guerra et al. and Goldstein et al. $(45,46)$, who reported that knockout of $G d f 11$ in either erythroid or hematopoietic lineages does not increase red cell production in transgenic mice nor in diverse models of anemia (45). These findings might be explained by a compensatory response to genetic deletion of GDF11 but are also consistent with the foregoing evidence that GDF11 is one of multiple SMAD2/3-pathway ligands collectively regulating erythropoiesis in mice. Together, these findings imply that SMAD2/3-pathway ligands act in concert to negatively regulate erythropoiesis in vivo and that sequestration of multiple ligands is necessary to explain the robust stimulation of red cell production by RAP536 (luspatercept) in normal and disease settings. This situation may be analogous to the field of skeletal muscle therapeutics, in which robust hypertrophy requires inhibition of more than one SMAD2/3-pathway ligand (47).

Preclinical studies with sotatercept or its analog RAP-011 corroborate findings obtained with luspatercept. In a murine model of $\beta$-thalassemia, RAP-011 treatment increased hemoglobin concentrations by promoting erythroid maturation (10). Additionally, RAP011 exerted beneficial effects on other clinicopathologic features of the disease, such as spleen size and iron overload, as evidenced by decreased serum iron levels and transferrin saturation (10).

\section{Clinical evaluation of activin receptor ligand traps}

Sotatercept was initially developed to increase bone mineral density in malignant bone disease or osteoporosis (48) but was found to increase red cell numbers in early human studies. Based on encouraging preclinical efficacy in raising red cell counts and hemoglobin concentrations, both luspatercept and sotatercept were assessed in phase II studies in patients with MDS (11, 12). A phase II, multicenter, open-label, dose-finding study of luspatercept (PACE-MDS) enrolled adult patients with MDS of low or intermediate risk (according to the International Prognostic Scoring System) or nonproliferative chronic myelomonocytic leukemia (white cell count $<13,000 / \mu \mathrm{L}$ ). Patients were classified as having low transfusion burden (defined as requiring fewer than 4 red blood cell units in the 8 weeks before treatment) or high transfusion burden (defined as requiring 4 or more red blood cell units in the 8 weeks before treatment) (11). Patients received luspatercept subcutaneously once every 21 days at dose concentrations ranging from 0.125 $\mathrm{mg} / \mathrm{kg}$ to $1.75 \mathrm{mg} / \mathrm{kg}$ body weight for 5 doses (over a maximum of 12 weeks). Patients in the expansion cohort were treated with $1.0 \mathrm{mg} / \mathrm{kg}$ luspatercept; dose titration up to $1.75 \mathrm{mg} / \mathrm{kg}$ was allowed, and patients could be treated with luspatercept for a maximum of 5 years. A total of 58 patients with MDS were enrolled, with 27 patients enrolled in the dose-escalation cohorts $(0.125-1.75 \mathrm{mg} / \mathrm{kg})$ and 31 patients in the expansion cohort (1.0-1.75 mg/kg). Thirty-two of 51 patients (63\%) receiving higher-dose luspatercept concentrations (0.75-1.75 mg/ 
$\mathrm{kg}$ ) achieved erythroid response versus 2 of 9 (22\%) receiving lower-dose concentrations (0.125-0.5 mg/kg). Independence from red blood cell transfusions was achieved in $38 \%$ of patients, and higher response rates were observed in MDS-RS (MDS associated with ring sideroblasts) and in patients with lower soluble EPO levels. Using similar criteria, sotatercept, in a phase II open-label dose-finding study in patients with low-risk or intermediate-1-risk MDS, showed reduction in transfusion burden in 36 of 74 (49\%) patients (12). This agent was also well tolerated without any major grade 3 or 4 adverse events.

Because of the effectiveness and tolerability of luspatercept in the foregoing phase II study and the narrower activity profile of luspatercept compared with sotatercept in preclinical studies, luspatercept was subsequently tested in MDS patients in a phase III, randomized, double-blind, placebo-controlled study (the MEDALIST trial) (49). Eligible patients displayed transfusiondependent anemia and MDS categorized as very-low-, low-, or intermediate-risk, as defined by the Revised International Prognostic Scoring System, with ringed sideroblasts. Enrolled patients $(n=226)$ were randomized 2:1 to receive either luspatercept, at a starting dose level of $1.0 \mathrm{mg} / \mathrm{kg}$ with titration up to 1.75 $\mathrm{mg} / \mathrm{kg}$ if needed, or placebo, subcutaneously every 3 weeks for at least 24 weeks. Of 153 patients receiving luspatercept, 58 (37.9\%) achieved the primary endpoint of red cell transfusion independence for at least 8 weeks compared with 10 of 76 patients (13.2\%) receiving placebo (odds ratio 5.1, $P<0.0001$ ). Of those receiving luspatercept, 43 of 153 (28.1\%) achieved the key secondary endpoint of red cell transfusion independence for at least 12 weeks (weeks 1-24) compared with 6 of 76 (7.9\%) receiving placebo (odds ratio 5.1, $P=0.0002$ ) (50). Erythroid hematologic improvement was achieved in 81 of 153 (52.9\%) patients receiving luspatercept versus 9 of 76 (11.8\%) patients receiving placebo during weeks 1 to 24 . The median duration of the longest single period of transfusion independence was 30.6 weeks for luspatercept versus 13.6 weeks for placebo. The most common treatment-associated adverse events of any grade included fatigue, diarrhea, asthenia, nausea, and dizziness and were mostly grade 1 or 2 in intensity.

Activin receptor ligand traps can also increase hemoglobin concentrations in patients with $\beta$-thalassemia. A recent phase III, randomized, double-blind, placebo-controlled study was conducted to determine the efficacy and safety of luspatercept in adult $\beta$-thalassemia patients requiring regular red cell transfusions. Patients were randomized 2:1 to receive either luspatercept, at a starting dose level of $1.0 \mathrm{mg} / \mathrm{kg}$ with titration up to $1.25 \mathrm{mg} / \mathrm{kg}$, or placebo, subcutaneously every 3 weeks for at least 48 weeks. A total of 336 patients were randomized, of whom 332 were treated. Forty-eight of 224 (21.4\%) patients in the luspatercept arm achieved the primary endpoint of at least $33 \%$ reduction in transfusion burden versus 5 of $112(4.5 \%)$ patients receiving placebo (odds ratio 5.79, $P<0.0001$ ) (51). These results in $\beta$-thalassemic patients are consistent with those in MDS patients as well as preclinical studies in disease models. Together, these findings indicate that activin receptor ligand traps act as erythroid maturation agents in alleviating ineffective erythropoiesis in multiple disease settings. These results have supported filing for regulatory approval of luspa- tercept in the United States and the European Union for both MDSs and $\beta$-thalassemia.

\section{Future prospects for activin receptor ligand traps}

Treatment of patients with MDS requires a complex and personalized variety of therapeutic approaches $(52,53)$. FDA-approved treatments for MDSs currently include the thalidomide analog lenalidomide and the DNA methyltransferase inhibitors azacytidine and decitabine, all of which provide partial effectiveness for anemia. Lenalidomide is approved for use in patients with deletion of chromosome $5 \mathrm{q}$, which occurs in approximately $10 \%$ of MDS cases. DNA methyltransferase inhibitors are approved for all subtypes of MDS but are predominantly used in intermediateand higher-risk cases. EPO mimetic agents are currently used for treating anemia associated with lower-risk MDS without deletion $5 \mathrm{q}$, but response rates are low and responses are generally not sustained. Thus, luspatercept has the potential to provide a therapeutic option for anemia in MDS patients, with a novel mechanism of action compared with other approved agents. While luspatercept previously demonstrated efficacy in MDS-RS, it is also currently being tested in clinical trials in other subtypes of MDS (COMMAND trial). Luspatercept has recently been approved in the United States as an erythroid maturation agent indicated for the treatment of anemia in adult patients with $\beta$-thalassemia who require regular red blood cell transfusions. This agent is also being tested in other hematologic diseases associated with anemia, such as myelofibrosis. In addition, luspatercept and sotatercept have displayed preclinical efficacy in diseases such as Diamond-Blackfan anemia and other anemic disorders $(7,9,54)$. It remains to be investigated in the clinic whether luspatercept could be used in combination with other approved agents for anemia to benefit from potentially complementary mechanisms of action (55).

\section{Conclusions}

Anemia is the most common cytopenia in patients with MDS. Research into mechanisms underlying ineffective erythropoiesis has revealed impaired erythroid differentiation in the majority of patients with anemia and identified this impairment as a strong prognostic factor for poor overall survival in MDSs. Although patients with MDS typically display elevated EPO concentrations indicative of a homeostatic response to hypoxia, these patients are paradoxically treated with erythropoiesis-stimulating agents as first-line therapy for anemia. Not surprisingly, the majority of such patients are nonresponders or develop resistance to these agents, which do not address the underlying impairment of erythroid maturation. As a consequence, patients are dependent on regular blood transfusions despite iron overloading and increased risk of other adverse outcomes. Together, these factors have prompted a search for alternative treatment options for anemia that can alleviate impaired erythroid differentiation in these patients.

TGF- $\beta$ superfamily signaling has emerged as an important regulator of erythropoiesis, including terminal erythroid maturation. Many patients with MDS exhibit overactive SMAD2/3 signaling in the bone marrow and abortive erythroid maturation. Inhibitors of SMAD2/3 signaling such the activin receptor ligand trap luspatercept have demonstrated promising ability to reduce anemia in preclinical and clinical studies. Additional 
clinical studies are under way to determine the extent to which luspatercept may improve anemia in MDS patients irrespective of mutation type, mutation number, or allelic burden.

\section{Acknowledgments}

Research conducted at Albert Einstein College of Medicine was funded by Leukemia Lymphoma Society, Evans Foundation, and the NIH to AV. Research conducted at Acceleron Pharma was funded by Acceleron.

Address correspondence to: Amit Verma, 1300 Morris Park Avenue, Albert Einstein College of Medicine, Montefiore Medical Center, Bronx, New York 10461, USA. Phone: 718.430.8761; Email: amit.verma@einstein.yu.edu.
1. Isufi I, et al. Transforming growth factor-beta signaling in normal and malignant hematopoiesis. JInterferon Cytokine Res. 2007;27(7):543-552.

2. Massagué J, Xi Q. TGF- $\beta$ control of stem cell differentiation genes. FEBS Lett. 2012;586(14):1953-1958.

3. Blank U, Karlsson S. TGF- $\beta$ signaling in the control of hematopoietic stem cells. Blood. 2015;125(23):3542-3550.

4. Naka K, et al. TGF- $\beta$-FOXO signalling maintains leukaemia-initiating cells in chronic myeloid leukaemia. Nature. 2010;463(7281):676-680.

5 . Mullen AC, Wrana JL. TGF- $\beta$ family signaling in embryonic and somatic stem-cell renewal and differentiation. Cold Spring Harb Perspect Biol. 2017;9(7):a022186.

6. Hata A, Chen YG. TGF- $\beta$ signaling from receptors to Smads. Cold Spring Harb Perspect Biol. 2016;8(9):a022061.

7. Suragani RN, et al. Transforming growth factor- $\beta$ superfamily ligand trap ACE-536 corrects anemia by promoting late-stage erythropoiesis. Nat Med. 2014;20(4):408-414.

8. Iancu-Rubin C, Mosoyan G, Wang J, Kraus T, Sung V, Hoffman R. Stromal cell-mediated inhibition of erythropoiesis can be attenuated by Sotatercept (ACE-011), an activin receptor type II ligand trap. Exp Hematol. 2013;41(2):155-166.e17.

9. Suragani RN, et al. Modified activin receptor IIB ligand trap mitigates ineffective erythropoiesis and disease complications in murine $\beta$-thalassemia. Blood. 2014;123(25):3864-3872.

10. Dussiot M, et al. An activin receptor IIA ligand trap corrects ineffective erythropoiesis in $\beta$ thalassemia. Nat Med. 2014;20(4):398-407.

11. Platzbecker U, et al. Luspatercept for the treatment of anaemia in patients with lower-risk myelodysplastic syndromes (PACE-MDS): a multicentre, open-label phase 2 dose-finding study with long-term extension study. Lancet Oncol. 2017;18(10):1338-1347.

12. Komrokji R, et al. Sotatercept with long-term extension for the treatment of anaemia in patients with lower-risk myelodysplastic syndromes: a phase 2, dose-ranging trial. Lancet Haematol. 2018;5(2):e63-e72.

13. Santini V. Treatment of low-risk myelodysplastic syndromes. Hematology Am Soc Hematol Educ Program. 2016;2016(1):462-469.

14. Heaney ML, Golde DW. Myelodysplasia. N Engl J Med.1999;340(21):1649-1660.

15. Camaschella C. Iron-deficiency anemia. $N$ Engl J Med. 2015;373(5):485-486.

16. Ali AM, et al. Severely impaired terminal erythroid differentiation as an independent prognostic marker in myelodysplastic syndromes. Blood Adv. 2018;2(12):1393-1402.

17. Zhao B, et al. Disruption of erythroid nuclear opening and histone release in myelodysplastic syndromes. Cancer Med. 2019;8(3):1169-1174.

18. Frisan E, et al. Defective nuclear localization of Hsp70 is associated with dyserythropoiesis and GATA-1 cleavage in myelodysplastic syndromes. Blood. 2012;119(6):1532-1542.

19. Schmierer B, Hill CS. TGF $\beta$-SMAD signal transduction: molecular specificity and functional flexibility. Nat Rev Mol Cell Biol. 2007;8(12):970-982.

20. Kemaladewi DU, et al. Cell-type specific regulation of myostatin signaling. FASEB J. 2012;26(4):1462-1472.

21. Bilandzic M, Stenvers KL. Betaglycan: a multifunctional accessory. Mol Cell Endocrinol. 2011;339(1-2):180-189.

22. Hill CS. Transcriptional control by the SMADs. Cold Spring Harb Perspect Biol. 2016;8(10):a022079.

23. Miyazawa K, Miyazono K. Regulation of TGF- $\beta$ family signaling by inhibitory Smads. Cold Spring Harb Perspect Biol. 2017;9(3):a022095.

24. Verma A, et al. Activation of the p38 mitogenactivated protein kinase mediates the suppressive effects of type I interferons and transforming growth factor- $\beta$ on normal hematopoiesis. J Biol Chem. 2002;277(10):7726-7735.

25. Mueller TD, Nickel J. Promiscuity and specificity in BMP receptor activation. FEBS Lett. 2012;586(14):1846-1859.

26. Antebi YE, et al. Combinatorial signal perception in the BMP pathway. Cell. 2017;170(6):1184-1196.e24.

27. Aykul S, Martinez-Hackert E. Transforming growth factor- $\beta$ family ligands can function as antagonists by competing for type II receptor binding. J Biol Chem . 2016;291(20):10792-10804.

28. Ning J, Zhao Y, Ye Y, Yu J. Opposing roles and potential antagonistic mechanism between TGF- $\beta$ and BMP pathways: implications for cancer progression. EBioMedicine. 2019;41:702-710.

29. Hinge A, Filippi MD. Deconstructing the complexity of TGF $\beta$ signaling in hematopoietic stem cells: quiescence and beyond. Curr Stem Cell Rep. 2016;2(4):388-397.

30. Blank U, Karlsson S. The role of Smad signaling in hematopoiesis and translational hematology. Leukemia. 2011;25(9):1379-1388.

31. Paulson RF, Shi L, Wu DC. Stress erythropoiesis: new signals and new stress progenitor cells. Curr Opin Hematol. 2011;18(3):139-145.

32. Borges L, Oliveira VKP, Baik J, Bendall SC, Perlingeiro RCR. Serial transplantation reveals a critical role for endoglin in hematopoietic stem cell quiescence. Blood. 2019;133(7):688-696.

33. Gao X, et al. TGF- $\beta$ inhibitors stimulate red blood cell production by enhancing selfrenewal of BFU-E erythroid progenitors. Blood. 2016;128(23):2637-2641.
34. Zhang H, et al. TGF- $\beta$ inhibition rescues hematopoietic stem cell defects and bone marrow failure in Fanconi anemia. Cell Stem Cell. 2016;18(5):668-681.

35. Joyce CE, et al. TGF $\beta$ signaling underlies hematopoietic dysfunction and bone marrow failure in Shwachman-Diamond Syndrome. JClin Invest. 2019;130:3821-3826.

36. Zhou L, et al. Inhibition of the TGF- $\beta$ receptor I kinase promotes hematopoiesis in MDS. Blood. 2008;112(8):3434-3443.

37. Zhou L, et al. Reduced SMAD7 leads to overactivation of TGF- $\beta$ signaling in MDS that can be reversed by a specific inhibitor of TGF- $\beta$ receptor I kinase. Cancer Res. 2011;71(3):955-963.

38. Blank U, et al. Smad7 promotes self-renewal of hematopoietic stem cells. Blood. 2006;108(13):4246-4254.

39. Bhagat TD, et al. miR-21 mediates hematopoietic suppression in MDS by activating TGF- $\beta$ signaling. Blood. 2013;121(15):2875-2881.

40. Velu CS, et al. Therapeutic antagonists of microRNAs deplete leukemia-initiating cell activity. J Clin Invest. 2014;124(1):222-236.

41. Santini V, et al. Phase 2 Study of the ALK5 inhibitor galunisertib in very low-, low-, and intermediate-risk myelodysplastic syndromes. Clin Cancer Res. 2019;25(23):6976-6985.

42. Sako D, et al. Characterization of the ligand binding functionality of the extracellular domain of activin receptor type IIb. J Biol Chem. 2010;285(27):21037-21048.

43. Richmond TD, Chohan M, Barber DL. Turning cells red: signal transduction mediated by erythropoietin. Trends Cell Biol. 2005;15(3):146-155.

44. Lin YW, Slape C, Zhang Z, Aplan PD. NUP98HOXD13 transgenic mice develop a highly penetrant, severe myelodysplastic syndrome that progresses to acute leukemia. Blood. 2005;106(1):287-295.

45. Guerra A, et al. Lack of $G d f 11$ does not improve anemia or prevent the activity of RAP-536 in a mouse model of $\beta$-thalassemia. Blood. 2019;134(6):568-572.

46. Goldstein JM, et al. Steady-state and regenerative hematopoiesis occurs normally in mice in the absence of GDF11. Blood. 2019;134(20):1712-1716.

47. Latres E, et al. Activin A more prominently regulates muscle mass in primates than does GDF8. Nat Commun. 2017;8:15153.

48. Raje N, Vallet S. Sotatercept, a soluble activin receptor type $2 \mathrm{~A}$ IgG-Fc fusion protein for the treatment of anemia and bone loss. Curr Opin Mol Ther. 2010;12(5):586-597.

49. Fenaux P, et al. Luspatercept in patients with lower-risk myelodysplastic syndromes. $\mathrm{NEnglJ}$ Med.2020;382(2):140-151.

50. Fenaux P, et al. The MEDALIST trial: results of a 
phase 3, randomized, double-blind, placebocontrolled study of luspatercept to treat patients with very low-, low-, or intermediate-risk myelodysplastic syndromes (MDS) associated anemia with ring sideroblasts (RS) who require red blood cell (RBC) transfusions. Blood. 2018;134(suppl 1):841.

51. Cappellini MD, et al. The BELIEVE trial: results of a phase 3, randomized, double-blind, placebocontrolled study of luspatercept in adult beta-thalassemia patients who require regular red blood cell (RBC) transfusions. Blood. 2018;132(suppl 1):163.

52. Platzbecker U. Treatment of MDS. Blood. 2019;133(10):1096-1107.

53. Fenaux P, Platzbecker U, Ades L. How we manage adults with myelodysplastic syndrome [published online ahead of print September 30, 2019]. Br J Haematol. https://doi.org/10.1111/bjh.16206.

54. Ear J, et al. RAP-011 improves erythropoiesis in zebrafish model of Diamond-Blackfan anemia through antagonizing lefty1. Blood. 2015;126(7):880-890.

55. Bewersdorf JP, Zeidan AM. Transforming growth factor (TGF) $\beta$ pathway as a therapeutic target in lower risk myelodysplastic syndromes. Leukemia. 2019;33(6):1303-1312.

56. Koury MJ, Haase VH. Anaemia in kidney disease: harnessing hypoxia responses for therapy. Nat Rev Nephrol. 2015;11(7):394-410. 